\title{
(a) \\ SINTAXONOMÍA DE LAS COMUNIDADES DE PLANTAS vasculares del sistema de drenaje del Alto Valle de Río Negro, Patagonia, Argentina
}

\author{
SYNTAXONOMY OF THE VASCULAR PLANTS COMMUNITIES OF THE DRAINAGE \\ system of the Alto Valle of Río Negro, Patagonia, Argentina
}

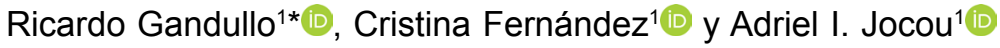

1. Departamento de Biología Aplicada. Facultad de Ciencias Agrarias, Universidad Nacional del Comahue. Río Negro, Argentina.

*rgandullo@yahoo.com.ar

Citar este artículo GANDULLO, R., C. FERNÁNDEZ \& A. I. JOCOU. 2019. Sintaxonomía de las comunidades de plantas vasculares del sistema de drenaje del Alto Valle de Río Negro, Patagonia, Argentina. Bol. Soc. Argent. Bot. 54: 567-587.

DOI: http://dx.doi. org/10.31055/1851.2372.v54. n4.24826

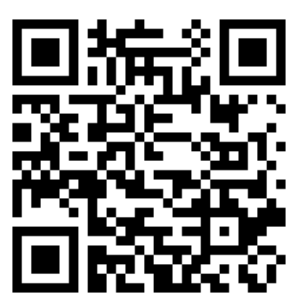

Recibido: 27 Junio 2019 Aceptado: 26 Agosto 2019 Publicado: 15 Diciembre 2019 Editor: Ramiro Aguilar (D)

ISSN versión impresa 0373-580X ISSN versión on-line 1851-2372

\section{SUMMARY}

Background and aims: The drainage system in the Alto Valle of Río Negro (Argentina), is integrated by a network of drains, and was designed to collect excess irrigation water from agricultural establishments. This artificial aquatic ecosystem allows the development of vegetal herbaceous species. The progress of urbanization towards agricultural lands increased the use of drains, due the discharge of industrial and urban effluents. The increase in non-agricultural effluents contaminate these environments producing changes in biota abundance and diversity. This work aims to propose the syntaxonomic categories of vegetation associated to the drainage system and determine the relevance of vegetation as a biological indicator in environmental impact studies.

M\&M: Floristic censuses obtained in previous vegetation studies were used and complemented with new surveys carried out during the period 2018-2019. Census were carried out with the phytosociological school of Zürich Montpellier methodology.

Results: The syntaxa found are described, according to the final phytosociological table interpretation. Its floristic, structural, syntaxonomic aspects and its potential as contamination bioindicator are discussed.

Conclusions: Twenty-eight syntaxonomical categories were established: three classes, five orders, five alliances, two sub-alliances and thirteen associations for the Alto Valle drainage system, organized according to three growth habits: floating, submerged and emerging aquatics. $80 \%$ of the associations have eutrophication bioindicator potential in the Alto Valle of Río Negro drainage system.

\section{KEY WORDS}

Aquatic communities, Argentina, Phytosociological taxonomy, urban and rural vegetation.

\section{RESUMEN}

Introducción y objetivos: En el Alto Valle de Río Negro (Argentina) el sistema de drenaje, integrado por una red de desagües, se diseñó para la captación del agua proveniente del excedente de riego de establecimientos agrícolas. Este ecosistema acuático artificial permite el desarrollo de especies vegetales de hábito herbáceo. El avance de la urbanización hacia los sectores agrícolas aumentó el uso de los desagües, a partir del vertido de efluentes industriales y urbanos. Esto establece el principal problema de contaminación en estos ambientes produciendo cambios en la diversidad y abundancia de la biota. Este trabajo tiene por objetivo establecer las categorías sintaxonómicas de la vegetación asociada al sistema de drenaje y determinar la pertinencia del uso de la vegetación como indicador biológico en los estudios de impacto ambiental.

M\&M: Se utilizaron censos florísticos obtenidos en estudios previos de vegetación y se complementaron con nuevos relevamientos realizados durante el periodo 2018-2019, con el uso de la metodología de la escuela fitosociológica de Zürich Montpellier.

Resultados: Se describen las sintaxa encontradas, a partir de la interpretación de la tabla fitosociológica final. Se discuten sus aspectos florísticos, estructurales, sintaxonómicos y su potencial bioindicador de contaminación.

Conclusiones: Se establecieron veintiocho categorías sintaxonómicas: tres clases, cinco ordenes, cinco alianzas, dos subalianzas y trece asociaciones para el sistema de drenaje del Alto Valle, ordenadas en función de tres hábitos de crecimiento: acuáticas flotantes, sumergidas y emergentes. El $80 \%$ de las asociaciones vegetales poseen potencial bioindicador de eutrofización en el sistema de drenaje del Alto Valle de Rio Negro.

\section{Palabras clave}

Argentina, comunidades acuáticas, taxonomía fitosociológica, vegetación urbana y rural. 


\section{INTRODUCCIÓN}

La contaminación tiende a ser el resultado de un proceso lento y acumulativo de la actividad humana. Todos los tipos de contaminación se caracterizan por alterar los ciclos naturales de materia y flujos de energía con repercusiones variadas, a veces irreversibles, en el funcionamiento de los ecosistemas. La mayoría de los parámetros utilizados para la evaluación de contaminación son de carácter físico-químico. Otro método de evaluación, es la utilización de organismos vivos, denominados indicadores biológicos o bioindicadores, que habitan ambientes contaminados con diferentes adaptaciones de tolerancia e intolerancia a distintas alteraciones del ambiente. Desde el punto de vista de la vegetación un indicador biológico es una especie o comunidad vegetal que brinda información sobre el impacto de ciertas prácticas antrópicas en el ambiente. Sintaxonómicamente, la asociación vegetal es la categoría básica del sistema de ordenamiento.

La asociación vegetal se caracteriza por tener determinadas cualidades mesológicas, una distribución geográfica concreta y una composición florística determinada (Rivas Martínez, 1987, 1995). La agrupación entre ciertas especies vegetales, y entre la vegetación y el medio, no es casual, sino causal. Esto permite analizar los nexos, enlaces o factores que determinan la coexistencia entre las distintas especies, fundamentalmente, a través de los inventarios fitosociológicos (Martínez Carretero et al., 2016). Alcaraz Ariza (2013) señala que la composición completa de especies de una asociación es la mejor expresión de las relaciones interespecíficas y con el ambiente. En este sentido, Ferreras \& Fidalgo (1999) indican que las especies se convierten en indicadores biológicos de su hábitat, de su medio y de otras especies.

Frente a un disturbio los organismos sensibles, que no soportan las nuevas condiciones impuestas, se comportan como intolerantes, mientras que otros, que son tolerantes, no se ven afectados. De modo que, variaciones inesperadas en la composición y estructura de las comunidades de organismos vivos pueden interpretarse como signos evidentes de algún tipo de contaminación
(Arocena \& Mazzeo, 1994; Alba Tercedor, 1996; Arocena et al., 2000).

La comunidad vegetal es mejor bioindicadora que las especies individuales de las condiciones ambientales (Ellenberg 1950, 1974; Ellenberg et al., 1992; Diekmann, 2003; Kollmann \& Fischer, 2003) y es considerada una importante herramienta en investigaciones ecológicas aplicadas y en proyectos de conservación y restauración (Kollmann \& Fischer, 2003; Fernández et al., 2018). Rodwell (1991, 1995) destaca la importancia de la fitosociología en el muestreo de hábitats y estudio de problemas ecológicos.

En este sentido autores como Venanzoni et al., (2006) y Ceschin et al., (2010) señalan a la asociación Potametum pectinati como bioindicadora de aguas con contaminación con materia orgánica. Por su parte, Finot y Ramírez (1996) indican que Hydrocotyletum ranunculoidis es una asociación bioindicadora de aguas contaminadas con residuos domésticos mientras que, la asociación Lemnetum gibbae es clara indicadora de eutrofización (Rivas Martínez, 1975).

Los biotopos urbanos y rurales, caracterizados por factores ecológicos extremos que dificultan el desarrollo de la vegetación nativa, son colonizados por comunidades sinantrópicas, en cuya composición florística las especies alóctonas y autóctonas cumplen un rol fundamental (Hruska, 1987; Jocou et al., 2018).

Varias de las comunidades acuáticas descritas para Chile (Oberdorfer, 1960; Ramírez \& San Martín, 1984, 2006; Ramírez et al., 1987; San Martín et al., 1993; San Martín et al., 1998; San Martín et al., 2001; Ramírez et al., 2004; Ramírez \& Álvarez, 2012) y para Patagonia (Conticello et al., 2002; Bezic et al., 2004; Fernández et al., 2018), están conformadas substancialmente por especies nativas (Jocou et al., 2018) y en menor medida por exóticas (principalmente europeas). $\mathrm{Si}$ bien las condiciones ecológicas y climáticas del norte patagónico exhiben características particulares, las comunidades acuáticas son similares en su composición florística, en mayor o menor grado, con las comunidades europeas.

En el Alto Valle de la provincia de Río Negro, el sistema de drenaje se diseñó para la captación del agua proveniente del excedente de riego 


\section{R. Gandullo et al. - Sintaxonomía de las comunidades de plantas vasculares}

de los establecimientos agrícolas. El conjunto de desagües, que integran la red de drenaje, constituye un ecosistema acuático artificial que permite el desarrollo tanto de hidrófitos como de helófitos, principalmente de hábito herbáceo. Sin embargo, el avance de la urbanización hacia los sectores agrícolas aumentó el uso de los desagües, con el vertido de efluentes industriales y urbanos (Lozeco, 2014). Esto establece el principal problema de contaminación en estos ambientes con los consecuentes cambios en la diversidad y abundancia de la biota (Fernández et al., 2018).

La fitosociología caracteriza la vegetación y reconoce su variación como respuesta a los cambios de los factores ecológicos. De ello resultan valiosos aportes para la evaluación de impacto ambiental, entre otros (Martínez Carretero et al., 2016). Este trabajo tiene por objetivo establecer la sintaxonomía de la vegetación del sistema de drenaje del Alto Valle de Río Negro y determinar la pertinencia del uso de la vegetación como indicador biológico en los estudios de impacto ambiental.

\section{Materiales y Métodos}

Área de estudio

El estudio se realizó en el sistema de drenaje del Alto Valle de Río Negro, ubicado entre los $66^{\circ} 45^{\prime}$ $\mathrm{E}$ y $68^{\circ} 30^{\prime} \mathrm{E}$ y los $38^{\circ} 30^{\prime} \mathrm{S}$ y $39^{\circ} 5^{\prime} \mathrm{S}$ con un área de $652 \mathrm{~km}^{2}$ (Jocou et al., 2018), en 88 desagües.

Se recurrió a los mapas del sistema de drenaje de Lozeco (2014) y los provistos por los consorcios de riego. Estos desagües, de ambientes urbanos y rurales, se caracterizan por ser dulceacuícolas lóticos de aguas someras, cuyas dimensiones no superar los 3 metros de profundidad y un ancho que oscila entre los 2 y 5 metros (Fig. 1). La disponibilidad de agua está condicionada a la funcionalidad del sistema de riego desde principios de septiembre a mayo. En los meses restantes se suspende el riego para tareas de mantenimiento en la red de riego y drenaje. Los desagües alojan comunidades vegetales acuáticas en forma continua o interrumpida, tanto temporal como espacialmente de acuerdo a la disponibilidad de agua.
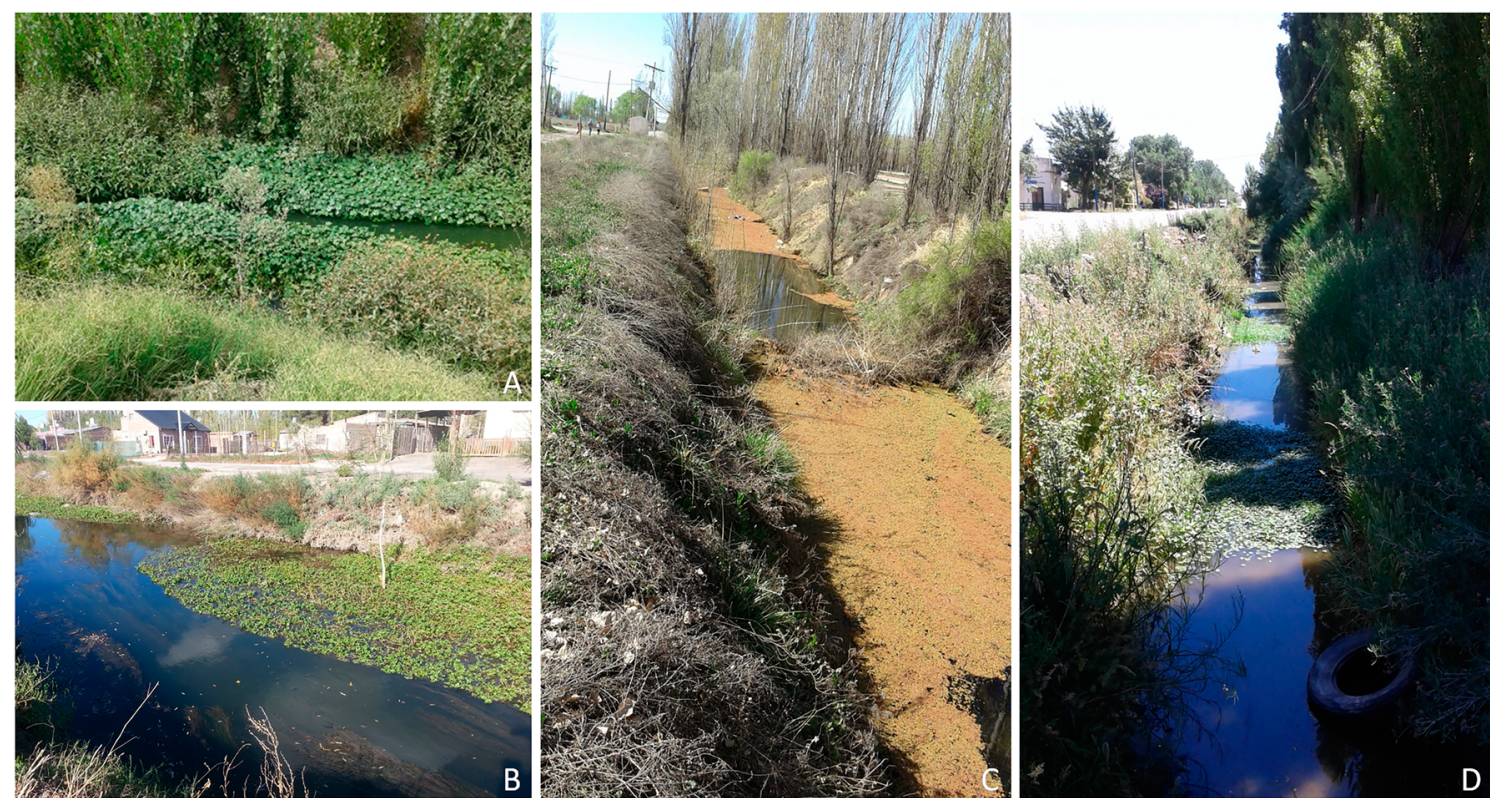

Fig. 1. Diversidad de desagües. A, D: de ambiente urbano, colonizado por la asociación Hydrocotyletum ranunculoidis. B: de ambiente urbano, colonizado por la comunidad de Ludwigia grandiflora ssp. hexapetala. C: de ambiente rural, colonizado con la asociación Azolletum filiculoidis. 


\section{Muestreo de la vegetación}

Se utilizaron los datos existentes de las comunidades vegetales acuáticas del Alto Valle de Río Negro de Fernández et al. (2018). Los mismos, se complementaron con nuevos relevamientos realizados durante el periodo 2018-2019. Los sitios de muestreos fueron seleccionados de acuerdo a su homogeneidad florística, fisonómica y ecológica (Roig, 1973), en superficies superiores al área mínima (Knapp, 1984) de $25 \mathrm{~m}^{2}$, cuyas especies se registraron con porcentajes de cobertura. La información fue sintetizada en un cuadro comparativo de la vegetación acuática que incluye el total de especies registradas con valores de grados de presencias y ordenadas en función del hábito de crecimiento de la especie dominante en: sumergida, flotante o emergente (Sculthorpe, 1967).

Para la nomenclatura y estatus de las especies se siguió el Catálogo de Plantas Vasculares del Cono Sur (IBODA, continuamente actualizado).

Los factores abióticos (turbidez del agua, velocidad y disponibilidad de luz) para cada sintaxa del cuadro comparativo sintético fueron obtenidos a partir de una escala graduada entre cero y tres (0-12-3) según Fernández et al. (2018). La profundidad de la columna de agua para cada desagüe se midió por medio de un bastón graduado con el sistema métrico decimal.

La nomenclatura sintaxonómica sigue el Código Internacional de Nomenclatura Fitosociológica (Weber et al., 2000).

\section{Análisis de datos}

Los datos de presencia de los taxones de la matriz original fueron analizados con el programa P-CORD (Mc Cune \& Mefford, 1999). La matriz se sometió a un análisis de clasificación mediante el método de la mínima varianza (Ward, 1963; Orloci, 1967) con el uso de la distancia euclídea como parámetro de similitud. El dendrograma final sirvió de base para la propuesta sintaxonómica (Müller-Dombois \& Ellenberg, 1974). Se utilizó la siguiente simbología para representar las categorías del esquema sintaxonómico: ${ }^{\circ}=$ clase; $*=$ orden; + $=$ alianza; $++=$ subalianza y los números arábigos = asociación.

Para la caracterización de las asociaciones del medio acuático se empleó la siguiente terminología: Pleustófitos: plantas acuáticas errantes, yacentes o suspendidas en agua y en ocasiones temporalmente enraizadas. Acropleustófitos: plantas cuyos órganos asimiladores flotan en la superficie del agua. Mesopleustófitos: cuyos órganos asimilables están suspendidos entre el fondo y la superficie. Pleustohelófitos: plantas que facultativamente, en las épocas desfavorables, radican en el fondo. Rizófitos: plantas acuáticas cuyas raíces o partes basales penetran en el sustrato, se incluyen aquí los carófitos, algunos briófitos y la mayoría de las vasculares acuáticas. Elodeidos: son rizófitos caulescentes con hojas sumergidas no divididas. Los órganos reproductores pueden o no alcanzar la superficie del agua. Miriofílidos: son rizófitos caulescentes con hojas divididas en finas lacinias, las partes reproductoras son emergentes, como ocurre en el género Myriophyllum. Ninfeidos y Batráquidos: son aquellas plantas que tienden a desarrollar formas terrestres, pertenecen a los géneros Ranunculus y Callitriche y que forman hojas flotantes y sumergidas. Reófilos: se aplica a las plantas que crecen en corrientes de aguas impetuosas.

\section{Resultados y Discusión}

La mayor parte de la flora de estos ambientes $(62,16 \%)$, comprende especies nativas, mientras que el 37,84 \% corresponde a especies exóticas. Las especies dominantes y codominantes representativas para cada una de las comunidades registradas son: Azolla filiculoides, Bolboschoenus maritimus, Elodea callitrichoides, Hydrocotyle ranunculoides, Lemna gibba, Ludwigia grandiflora ssp. hexapetala, Myriophyllum aquaticum, Nasturtium officinale, Phragmites australis, Stuckenia pectinata, Typha angustifolia, T. domingensis, T. latifolia y T. subulata.

En el dendrograma de los censos de vegetación (Fig. 2), surgen tres grupos relacionados con el hábito de crecimiento de la flora acuática del Alto Valle. El primer grupo, está conformado por tres comunidades sumergidas $(\mathrm{C} 1, \mathrm{C} 2$ y $\mathrm{C} 3)$ cuya afinidad florística sugiere que poseen requerimientos de sitios parecidos. El segundo grupo (comunidades C6, C7, $\mathrm{C} 10, \mathrm{C} 11, \mathrm{C} 13, \mathrm{C} 8, \mathrm{C} 12, \mathrm{C} 9$ y C14) presenta una vegetación más diversa y conformado por especies emergentes con alturas variables. El tercer grupo, representado por dos comunidades caracterizadas por especies flotantes ( $\mathrm{C} 4$ y $\mathrm{C} 5$ ), evidencia escasa afinidad con el primero y segundo grupo. 


\section{R. Gandullo et al. - Sintaxonomía de las comunidades de plantas vasculares}

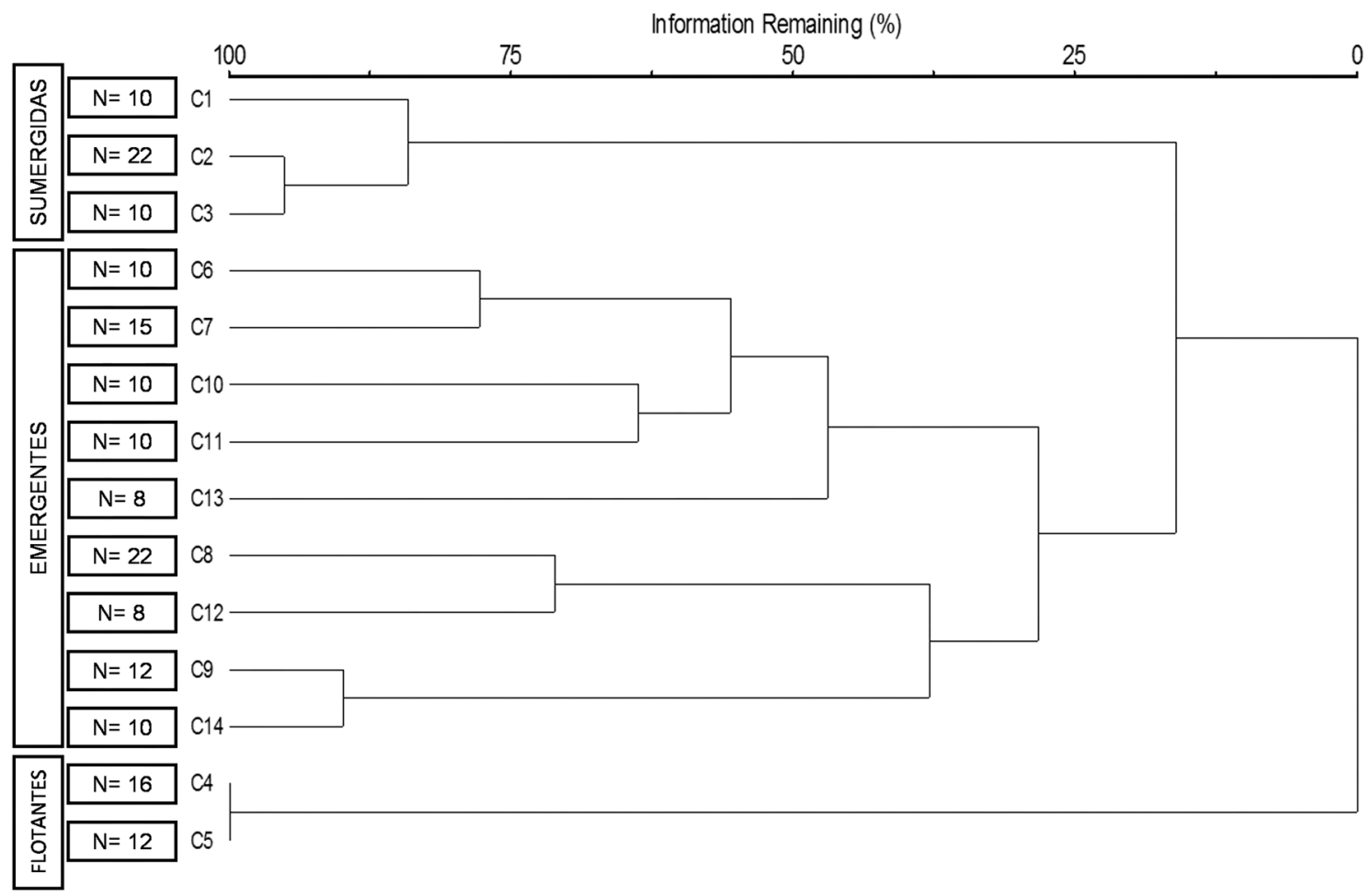

Fig. 2. Dendrograma de los catorce grupos florísticos de la vegetación del sistema de drenaje del Alto Valle de Río Negro. Referencias= C1: Elodeetum callitrichoidis; C2: Potametum pectinati; C3: Myriophylletum aquaticum; C4: Azolletum filiculoidis; C5: Azollo filiculoides - Lemnetum gibbae; C6: Hydrocotyletum ranunculoidis; C7: Nasturtietum officinalis; C8: Typhetum domingensis; C9: Typhetum angustifoliae; C10: Typhetum subulatae; C11: Typhetum latifoliae; C12: Bolboschoenetum maritimi; C13: Comunidad de Ludwigia grandiflora ssp. hexapetala; C14: Phragmitetum australis; N: número de relevamientos de cada grupo florístico.

Los tres grupos fueron analizados comparativamente (Tabla 1) para la clasificación jerárquica, lo cual permite la confección del esquema sintaxonómico. El mismo se caracteriza por trece asociaciones, una comunidad vegetal, cinco alianzas, dos subalianzas, cinco órdenes y tres clases. Se distribuyen y ordenan en función de tres hábitos de crecimiento: acuática flotante, sumergida y emergente de acuerdo a la especie dominante.

Esquema sintaxonómico:

Formación: Vegetación acuática flotante libre

${ }^{\circ}$ Lemnetea minoris W. Koch \& R. Tx. In R. Tx. 1955
* Lemnetalia gibbae Landolt 1999

+ Azollo filiculoides -Lemnion gibbae Landolt 1999

1. Azolletum filiculoidis ass. nov.

2. Azollo filiculoides-Lemnetum gibbae ass. nov.

Formación: Vegetación acuática sumergida

${ }^{\circ}$ Potametea Klika in Klika \& Novák 1941

* Potametalia pectinati Koch 1926

+ Potamion pectinati (Koch 1926) Görs 1977

1. Potametum pectinati Cartensen 1955

2. Myriophylletum aquaticum Medina 1988

* Parvopotametalia Den Hartog \& Segal 1964

+ Parvopotamion Vollmar 1947

1. Elodeetum callitrichoidis ass. nov. 
Formación: Vegetación acuática emergente

- Magnocari elatae - Phragmitetea australis Klika in Klika \& Novák 1941 nom. inv. Rivas Martínez \& col. 2011

* Phragmitetalia australis Koch 1926 em. Pignatti 1954

+ Phragmition australis Koch 1926 nom. mut.

Rivas Martínez y col. 2011

++ Phragmitenion australis (Koch 1926)

Rivas Martínez in Rivas Martínez, Costa, Castroviejo \& E. Valdés 1980

1. Phragmitetum australis Soó 1927 nom. mut. propos. Schmale 1939

2. Typhetum angustifoliae Pignatti 1953

3. Typhetum latifoliae Nowinski 1930

4. Typhetum domingensis Brullo et al. 1994

5. Typhetum subulatae ass. nov.

++ Bolboschoenenion maritimi Rivas

Martínez in Rivas Martínez, Costa, Castroviejo \& E. Valdés 1980 nom. mut. Rivas \& col. 2011

1. Bolboschoenetum maritimi $\mathrm{Br}$. Bl. 1931

* Nasturtio officinalis - Glycerietalia fluitantis Pignatti 1953

+ Apion nodiflori Segal in Westhoff \& den Held 1969

1. Nasturtietum officinalis (Seibert 1962) Oberd. et al. 1967

2. Hydrocotyletum ranunculoidis Corbetta \& Lorenzoni 1976

3. Comunidad de Ludwigia grandiflora ssp. hexapetala

Descripción de las comunidades vegetales acuáticas

La vegetación acuática del Alto Valle de Río Negro está constituida por catorce comunidades que pueden adscribirse a tres clases Lemnetea minoris, Potametea y Magnocari elatae - Phragmitetea australis.

\section{Vegetación acuática flotante libre}

${ }^{\circ}$ Lemnetea minoris W. Koch \& R. Tx. in R. Tx. 1955

Clase de distribución cosmopolita que agrupa a la vegetación dulceacuícola no enraizada constituida por acropleustófitos y mesopleustófitos de pequeña talla como las lentejas de agua, formadas por: Lemna minor, L. gibba, L. trisulca, Spirodela polyrrhiza, Wolffia spp. y Wolffiella spp. o los hidropteridales como Azolla filiculoides y Salvinia natans. Todos los tipos de vegetación incluidos en la clase, salvo
Lemnetum gibbae de aguas contaminadas o Azolletum carolinianae de los arrozales, son muy sensibles a la acción antropogénica.

De acuerdo con la tipología propuesta por Landolt (1999) para la clase Lemnetea minoris, reconoce separar la vegetación en tres órdenes según la presencia de especies comunes pero restringidas geográfica y climáticamente; de esta manera la clase Lemnetea se divide en tres órdenes.

- Lemnetalia minoris: tipo de clima fresco y continental cálido (Lemna minor y L. turionifera);

- Lemnetalia gibbae: tipo de clima oceánico templado a subtropical (Lemna gibba, L. obscura, L. japonica y Wolffia globosa);

- Lemnetalia aequinoctialis: tipo de clima subtropical a tropical (Wolffiella oblonga y Limnobium laevigatum)

Las comunidades flotantes aquí descriptas se encuentras representada en el orden Lemnetalia gibbae y las alianzas Azollo filiculoides - Lemnion gibbae y Lemnion gibbae (no incluida en el esquema sintaxonómico).

\section{* Lemnetalia gibbae Landolt 1999}

De acuerdo a Landolt (1999), el orden se distribuye de manera similar a las especies características, que habitan aguas quietas y vertientes lentas, de oligotróficas hasta eutróficas, de regiones templadas hasta subtropicales de todo el mundo (excepto Australia). En Argentina, Lemna gibba se encuentra distribuida entre 0 y $4000 \mathrm{~m}$ de altitud, a excepción del nordeste y del extremo austral (Landolt, 1996).

En América del sur este orden se encuentra representado por una alianza y dos asociaciones.

Especie característica: Lemna gibba.

+ Azollo filiculoides - Lemnion gibbae Landolt 1999

La alianza está restringida a regiones de Sudamérica que son relativamente frescas con inviernos suaves, también se encuentra en Europa debido a la introducción de las especies Azolla filiculoides y Lemna minuta.

La colonización de aguas comienza con Lemna gibba (aguas ricas en nutrientes), L. minuta (aguas menos eutróficas) o Azolla spp. (aguas inicialmente pobres en nitrógeno). L. valdiviana y Wolffiella oblonga solo están presentes en situaciones de más estabilidad. En etapas posteriores, ambas especies 
R. Gandullo et al. - Sintaxonomía de las comunidades de plantas vasculares

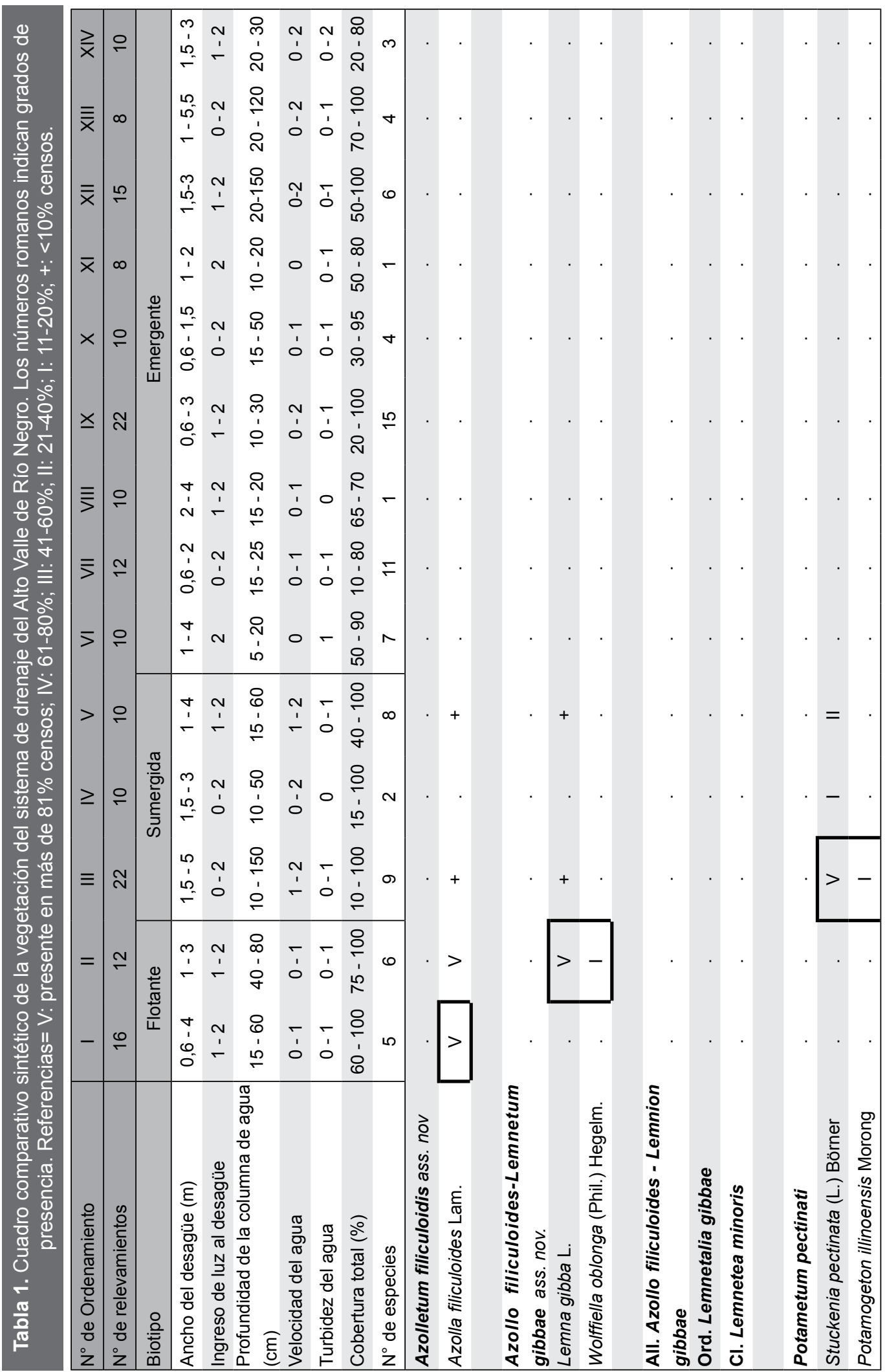


Bol. Soc. Argent. Bot. 54 (4) 2019

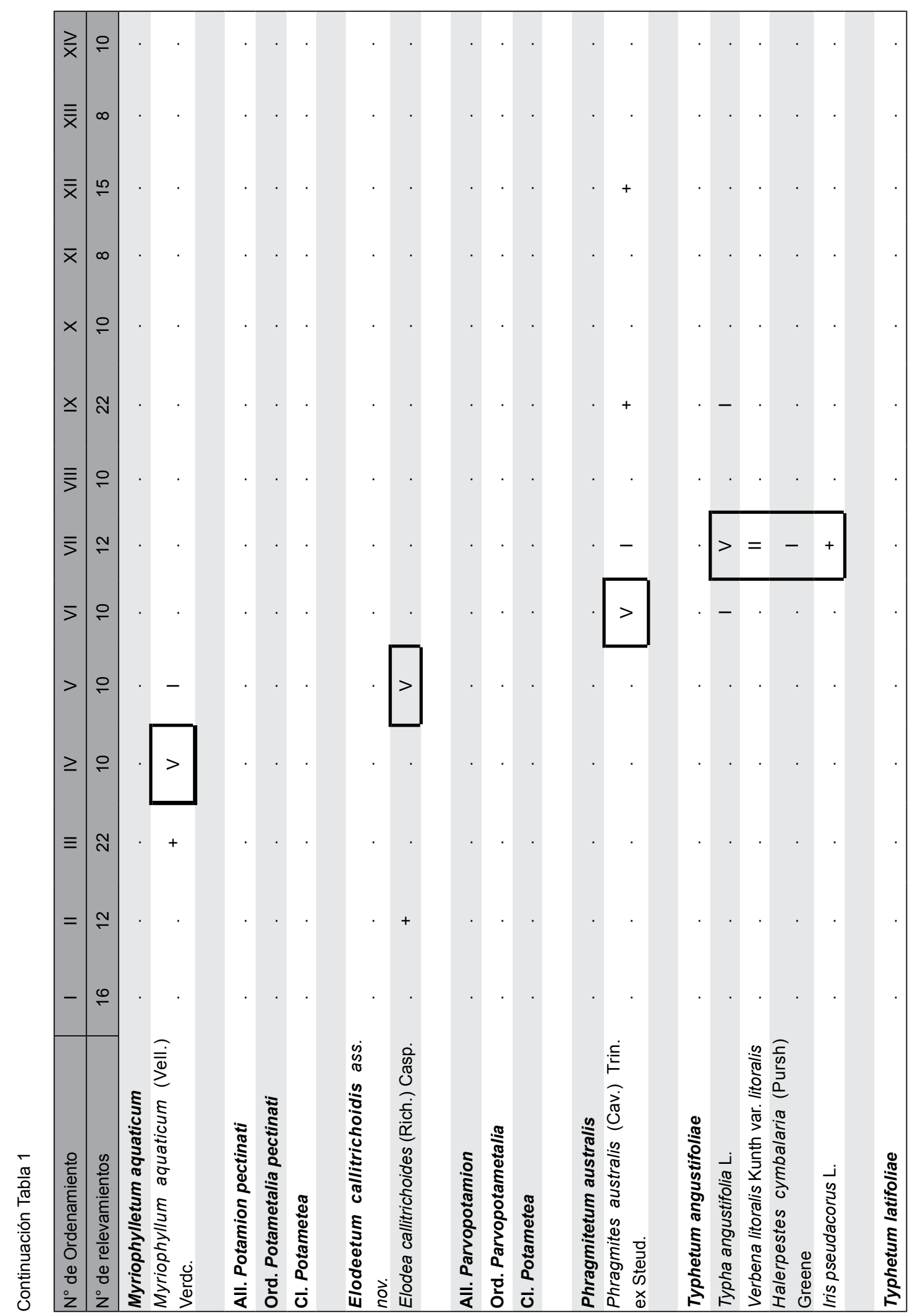


R. Gandullo et al. - Sintaxonomía de las comunidades de plantas vasculares

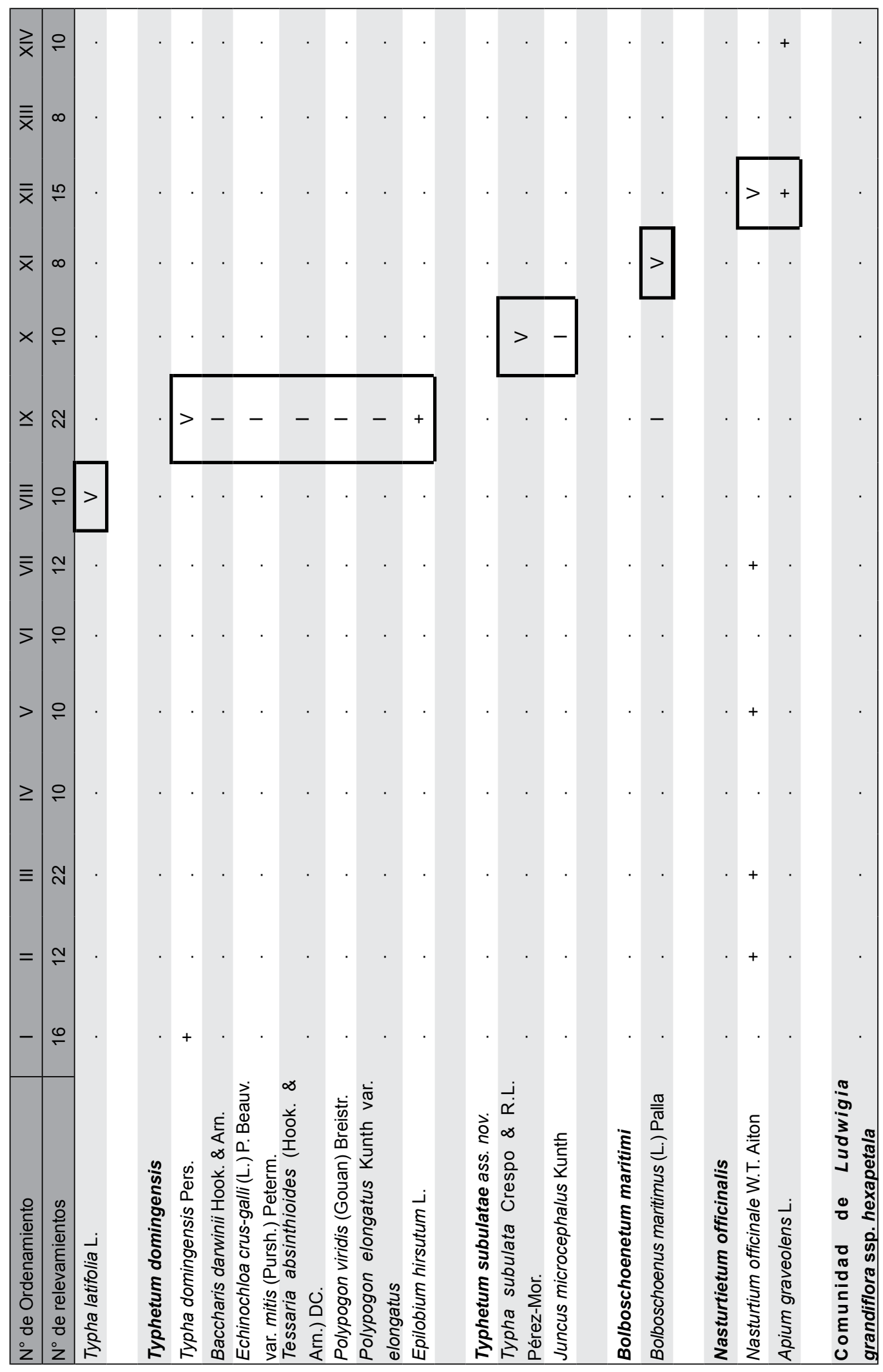


Bol. Soc. Argent. Bot. 54 (4) 2019

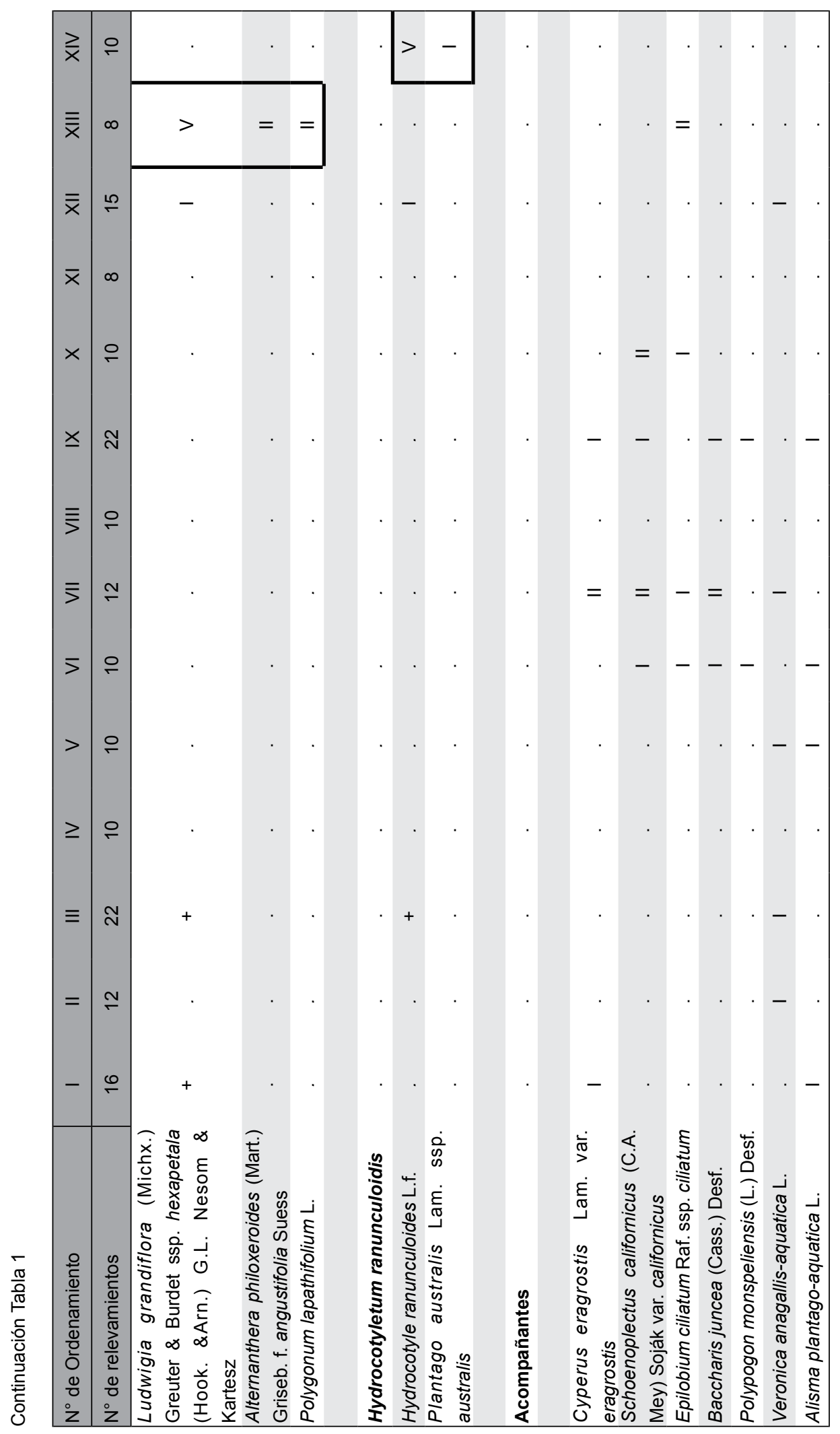




\section{R. Gandullo et al. - Sintaxonomía de las comunidades de plantas vasculares}

forman una densa segunda capa sumergida debajo de la superficie. En esta situación, L. gibba ya no puede formar capas compactas.

La asociación tipo de la alianza es Lemno minutae - Lemnetum gibbae Landolt 1999 cuya especie característica es Azolla filiculoides y especies frecuentes Lemna gibba y L. minuta.

1. Azolletum filiculoidis ass. nov. (Tabla 1, orden I y Tabla 2)

Typus nomenclatorius associationis: Tabla 2, inv. 90

Diagnosis. Asociación caracterizada básicamente por Azolla filiculoides (Fernández et al., 2018) muy pobre florísticamente, debido a su alta densidad que bloquea el ingreso efectivo de la radiación fotosintéticamente activa y ocasiona la muerte de las especies sumergidas. Indicadora de medio eutrofizado con disponibilidad de nitrógeno combinado, de origen abiótico (síntesis química de fertilizantes) o por la acción de microrganismos (fijación biológica de nitrógeno) con disponibilidad de fósforo para su óptimo crecimiento. Común en desagües con alta heliofanía y con efluentes urbanos que contienen residuos domésticos.

Especie característica: Azolla filiculoides.
2. Azollo filiculoides - Lemnetum gibbae ass. nov. (Tabla 1, orden II y Tabla 3)

Typus nomenclatorius associationis: Tabla 3, inv. 80

Diagnosis. Asociación nueva para la ciencia, dominada por Azolla filiculoides y Lemna gibba, común en el sistema de drenaje y considerada indicador de ambientes acuáticos eutrofizados (Fernández et al., 2018). Este tipo de vegetación se desarrolla entre inicios de primavera a finales de otoño, pero particularmente entre enero y marzo. En los desagües del Alto Valle, la abundancia es directamente proporcional al grado de eutrofización. En este período Lemna gibba muestra su evidente gibosidad, un rasgo característico que facilita la identificación tanto para la especie como para la asociación. Sin embargo, durante la primavera y la primera etapa del verano la gibosidad a veces está ausente y la especie puede confundirse fácilmente con Lemna minor (Landolt, 1980; Štěpánková et al., 2010).

Si bien, la nueva asociación propuesta Azollo filiculoides - Lemnetum gibbae, presenta el mismo comportamiento que Lemno Azolletum filiculoidis Oberd. 1960, muy abundante en lagunas mediterráneas del territorio chileno (San

Tabla 2. Azolletum filiculoidis Gandullo R., Fernández C. y Jocou A. I. Typus nomenclatorius associationis: inventario 90 (Gandullo, Fernández y Jocou) Prov. de Río Negro: Gral. Roca, zona urbana (Barrio

Belgrano) 18-03-2017. Densidad de especies: 1 (rango:1-3). Referencias= Localidades, CC: Contralmirante Cordero; Ce: Cervantes; GFO: General Fernández Oro; A: Allen; Ci: Cipolletti; GR: General Roca; Ma: Mainqué. Valores de cobertura, +: (<1); 1: (1-10); 2: (11-25); 3: (26-50); 4: (51-75); 5: (>75).

\begin{tabular}{|c|c|c|c|c|c|c|c|c|c|c|c|c|c|c|c|c|}
\hline Número de ordenamiento & 1 & 2 & 3 & 4 & 5 & 6 & 7 & 8 & 9 & 10 & 11 & 12 & 13 & 14 & 15 & 16 \\
\hline Número de censo & 94 & 110 & 90 & 250 & 87 & 79 & 4 & 128 & 126 & 10 & 105 & 104 & 37 & 5 & 257 & 9 \\
\hline Localidad & $\mathrm{Ce}$ & $\mathrm{Ma}$ & GR & $\mathrm{Ci}$ & $A$ & CG & GFO & $\mathrm{Ce}$ & $\mathrm{Ce}$ & $\mathrm{Ci}$ & $\mathrm{Ma}$ & $\mathrm{Ma}$ & $\mathrm{CC}$ & GFO & $\mathrm{Ci}$ & $\mathrm{Ci}$ \\
\hline Cobertura total $\%$ & 100 & 100 & 100 & 100 & 100 & 98 & 91 & 90 & 80 & 80 & 80 & 70 & 60 & 40 & 30 & 20 \\
\hline Número de especies & 1 & 1 & 1 & 1 & 1 & 1 & 2 & 1 & 1 & 2 & 3 & 3 & 1 & 1 & 2 & 1 \\
\hline \multicolumn{17}{|l|}{ Azolletum filiculoidis } \\
\hline Azolla filiculoides & 5 & 5 & 5 & 5 & 5 & 5 & 5 & 5 & 5 & 5 & 5 & 4 & 4 & 3 & 3 & 2 \\
\hline Alisma plantago-aquatica & . & . & . & . & . & . & . & . & . & . & + & 1 & . & . & . & . \\
\hline $\begin{array}{l}\text { Cyperus eragrostis var. } \\
\text { eragrostis }\end{array}$ & . & . & . & . & . & $\cdot$ & . & $\cdot$ & $\cdot$ & $\cdot$ & + & + & . & . & . & . \\
\hline Typha domingensis & . & . & . & . & . & . & + & . & . & . & . & . & . & . & + & . \\
\hline $\begin{array}{l}\text { Ludwigia grandiflora ssp. } \\
\text { hexapetala }\end{array}$ & . & . & & . & . & . & & . & . & + & & . & . & . & . & . \\
\hline
\end{tabular}




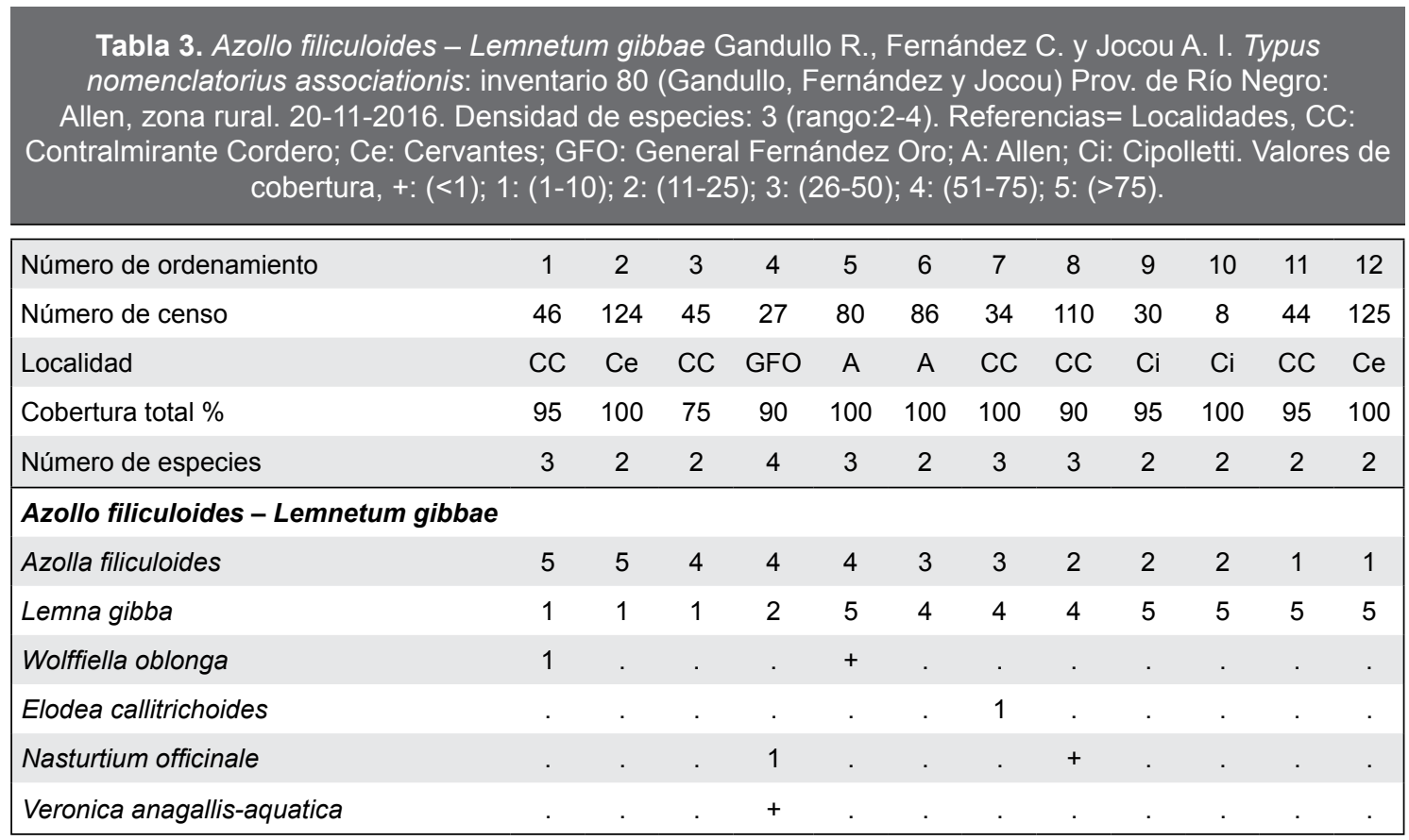

Martín et al., 2003; Ramírez et al., 2014), difiere florísticamente de la asociación chilena por tener Lemna gibba como especie característica de la asociación conjuntamente con Azolla filiculoides.

Especies características: Azolla filiculoides y Lemna gibba.

+ Lemnion gibbae R. Tx. \& Schwabe in R. Tx. 1974

Presenta asociaciones con un solo estrato de acropleustófitos fácilmente desplazables por el viento, que forman parte del epipleon de aguas neutras a básicas ( $\mathrm{pH}$ 6,6-9,2), eutrofizadas, ricas en iones $\left(\mathrm{PO}_{4}^{-3} 2,0-4,2 \mathrm{mg} / 1 ; \mathrm{NO}_{3}^{-} 6,0\right.$ $18,0 \mathrm{mg} / \mathrm{l}) \mathrm{y}$ frecuentemente contaminadas con microorganismos.

Especie característica Lemna gibba.

A la vista de los aportes de Landolt (1999) se correspondería ubicar las sintaxa Lemnion gibbae y Lemnetum gibbae en el orden Lemnetalia gibbae y no en Lemnetalia minoris.

Hasta el momento, la asociación Lemnetum gibbae ha sido identificada con pocos relevamientos en los canales de desagüe del Alto Valle de Río Negro. Posiblemente esto se deba a que los vertidos orgánicos e inorgánicos no logren una suficientemente concentración de nutrientes para estabilizar la comunidad. No obstante, no se descarta su establecimiento y por lo tanto se ha decidido describirla sin incluirla en el esquema sintaxonómico.

1. Lemnetum gibbae (W. Koch 1954) Miyawaki \& R. Tx. 1960

Asociación constituida por Lemna gibba, acropleustófito cosmopolita, de aguas dulces eutróficas a hipertróficas, estancadas o de escaso caudal, en general con abundante materia orgánica, amplia disponibilidad de luz y con cierta tolerancia a salinidad. Forma parte del epipleon de aguas permanentes, remansadas, eutrofizadas y ricas en iones. Se presenta en forma de poblaciones prácticamente monoespecíficas de Lemna gibba, que llegan a cubrir por completo la superficie del agua. Es muy tolerante a altas concentraciones de nitrógeno, fósforo, calcio y cloruros (Scoppola, 1982; Šumberová, 2011) de diversos orígenes, entre ellos los fertilizantes utilizados en los cultivos. 


\section{R. Gandullo et al. - Sintaxonomía de las comunidades de plantas vasculares}

Vegetación acuática sumergida

${ }^{\text {o }}$ Potametea Klika in Klika \& V. Novák 1941

La clase Potametea incluye la vegetación arraigada, de aguas dulces o con moderada mineralización, constituida por plantas acuáticas de diversos tamaños que colonizan las aguas permanentes y profundas o las estacionales y someras de ríos, arroyos, lagunas y charcas. Las diferentes comunidades que se reconocen en esta clase responden a la distinta naturaleza de las aguas que colonizan (eutrofia, físico-química, profundidad, permanencia, intensidad de la corriente, entre otras). Esta clase agrupa comunidades acuáticas de distribución cosmopolita muy abundante y bastante homogéneas en todo el mundo, formada por hidrófitos enraizantes sobre todo perennes (Potametalia pectinati) o por mesopleustófitos: utriculáridos y ceratofílidos, suspendidos entre el fondo y la superficie (Utricularietalia vulgaris).

Entre las especies constantes de la clase están Ceratophyllum demersum, Potamogeton natans, P. lucens y Stuckenia pectinata, siendo esta última la más representativa para nuestra zona de estudio.

En el Alto Valle de Río Negro reconocemos dos órdenes, dos alianzas y tres asociaciones:

\section{* Potametalia pectinati Koch 1926}

En este orden se incluyen aquellas comunidades de agua dulce, ricas o pobres en nutrientes, de distribución cosmopolita y formadas por hidrófitos enraizantes (rizófitos): elodeidos, miriofílidos, ninfeidos y batráquidos (Castro González, 2015).

\section{+ Potamion pectinati (Koch 1926) Görs 1977}

Alianza de asociaciones dulceacuícolas fluvio-lacustres de elodeidos y miriofílidos de aguas lénticas, lóticas o reófilas, de distribución cosmopolita. En los medios estudiados reconocemos las siguientes comunidades:

\section{Potametum pectinati Cartensen 1955}

Esta asociación suele ser monospecífica o compuesta por pocas especies. La especie dominante es Stuckenia pectinata, una macrófita sumergida enraizada que se encuentra en cuerpos de agua eutróficos e hipertróficos, de cierta profundidad, caracterizada a menudo por una alta turbidez, condiciones anóxicas y a veces alta concentración de sales (Landucci et al., 2011). Esta asociación es un indicador de la mala calidad del agua debido a la contaminación orgánica (Avena et al., 1980; Baldoni \& Biondi, 1993; Venanzoni \& Gigante, 2000; Venanzoni et al., 2006; Ceschin et al., 2010).

En nuestra región, durante las estaciones primavera-verano, aparece la comunidad de Stuckenia pectinata en abundancia y con tupido crecimiento de largos tallos que frecuentemente superan los $2 \mathrm{~m}$. Se presenta en desagües de aguas eutróficas de corriente lenta a media, con una profundidad que varía de 1 a $3 \mathrm{~m}$. Su desarrollo y establecimiento se ve limitado por la cobertura de comunidades flotantes, debido a la competencia lumínica, como consecuencia no pueden coexistir (Akasaka \& Takamura, 2011).

El tratamiento sintaxonómico de las comunidades de Stuckenia pectinata, ha sido controvertido, consideramos acertada su inclusión en el orden Potametalia pectinati, alianza Potamion pectinati como indica Castro González (2015).

\section{Myriophylletum aquaticum Medina 1988}

Asociación descripta y considerada nativa para Chile por González López (2005). Esta comunidad es casi pura, dominada por Myriophyllum aquaticum con alta cobertura y escasa riqueza florística, al igual que su par en Chile. En nuestra área de estudio es preferente de sustratos fangosos con alto contenido orgánico, aguas someras de escurrimiento lento. No tolera aguas salobres y requiere mucha luz. Presenta distribución en las regiones este y norte de argentina.

\section{* Parvopotametalia Den Hartog \& Segal 1964}

En el orden se incluyen las comunidades de pequeñas plantas acuáticas (miriofílidos, elodeidos y ranúnculos) que se desarrollan en aguas someras estacionales o semipermanentes, dulces o subsalinas con diferentes grados de eutrofia. Las comunidades que se incluyen en este orden suelen ser pioneras en cuerpos de agua con dimensiones reducidas y en el caso de charcas estacionales constituyen comunidades estables que recolonizan dichas depresiones en años sucesivos siempre que el volumen de agua retenido lo permita. La escasez de lluvias primaverales restringe el desarrollo de estas asociaciones que pueden originar una biomasa importante y rellenar las pequeñas concavidades que son un tipo de hábitat acuático ciertamente interesante. El orden presenta dos alianzas que se separan de acuerdo a la distinta composición 
florística de sus comunidades. En el Alto Valle distinguimos a:

\section{+ Parvopotamion Vollmar 1947}

En esta alianza se incluyen las asociaciones caracterizadas por la ausencia de ranúnculos acuáticos y la abundancia de elodeidos, en particular de los géneros Potamogeton y Zannichellia, con ambientes de mayor régimen hídrico.

1. Elodeetum callitrichoidis ass. nov. (Tabla 1, orden V y Tabla 4).

Typus nomenclatorius associationis: Tabla 4, inv. 260

Diagnosis. Elodea callitrichoides es la especie dominante de la comunidad con altos porcentajes de cobertura, desde $45 \%$ hasta $95 \%$, relativamente común en las localidades del Alto Valle Río Negro. Cumple mayoritariamente su ciclo bajo el agua a excepción cuando florece donde sus pequeñas flores blancas flotan encima de la superficie. Prefiere suaves corrientes de agua dulce oligotrófica o algo eutrofizadas, con baja o nula turbidez y de baja profundidad $(40-60 \mathrm{~cm})$. Esta comunidad es acompañada en su composición florística y estructura por otras macrófitas sumergidas como Myriophyllum aquaticum y Stuckenia pectinata, especies características de otras asociaciones de la misma clase. Se establece en desagües cercanos a establecimientos frutícolas (Fernández et al., 2018). En el área de estudio se reconoce fácilmente por sus flores blancas que emergen del agua en la época de reproducción.

Especies características: Elodea callitrichoides.

- Magnocari elatae - Phragmitetea australis Klika in Klika \& Novák 1941 nom. inv. Rivas Martínez $\&$ col. 2011

Vegetación hidrófila enraizada e higroturbosa perenne, constituida por grandes helófitos erguidos o decumbentes o hierbas acuosas helofíticas, propia de los bordes de cursos de aguas someras, dulces o levemente salobres, de lagunas y zonas pantanosas que sufren anegamiento permanente o durante la mayor parte del año. Aparece en medios desde oligo a eutróficos, fundamentalmente en la zona templada del hemisferio boreal, aunque algunas comunidades llegan a ser cosmopolitas (Molina, 1996). En el

Tabla 4. Elodeetum callitrichoidis Gandullo R., Fernández C. y Jocou A. I. Typus nomenclatorius associationis: inventario 260 (Gandullo, Fernández y Jocou) Prov. de Río Negro: Cipolletti, zona rural. 18-03-2017 Densidad de especies: 2 (rango:1-5). Referencias= Localidades, Ce: Cervantes; Ci: Cipolletti; CS: Cinco Saltos; VR: Villa Regina; GG: General Godoy. Valores de cobertura, +: $(<1)$; 1 : $(1$ 10); 2: (11-25); 3: (26-50); 4: (51-75); 5: (>75).

\begin{tabular}{|c|c|c|c|c|c|c|c|c|c|c|}
\hline Número de ordenamiento & 1 & 2 & 3 & 4 & 5 & 6 & 7 & 8 & 9 & 10 \\
\hline Número de censo & 28 & 146 & 53 & 260 & 7 & 97 & 140 & 130 & 98 & 127 \\
\hline Localidad & $\mathrm{Ci}$ & VR & CS & $\mathrm{Ci}$ & $\mathrm{Ci}$ & $\mathrm{Ce}$ & VR & GG & $\mathrm{Ce}$ & $\mathrm{Ce}$ \\
\hline Cobertura total $\%$ & 97 & 95 & 94 & 90 & 92 & 80 & 65 & 60 & 40 & 40 \\
\hline Número de especies & 5 & 5 & 4 & 4 & 4 & 2 & 1 & 1 & 1 & 2 \\
\hline \multicolumn{11}{|l|}{ Elodeetum callitrichoidis } \\
\hline Elodea callitrichoides & 5 & 5 & 5 & 5 & 4 & 4 & 4 & 4 & 3 & 3 \\
\hline Azolla filiculoides & + & . & . & . & . & . & . & . & . & + \\
\hline Lemna gibba & + & . & . & . & 1 & . & . & . & . & . \\
\hline Myriophyllum aquaticum & + & + & 1 & . & . & . & . & . & . & . \\
\hline Nasturtium officinale & . & + & + & . & . & . & . & . & . & . \\
\hline Stuckenia pectinata & + & . & . & + & 2 & 1 & . & . & . & . \\
\hline Veronica anagallis-aquatica & . & + & . & + & + & . & . & . & . & . \\
\hline Alisma plantago-aquatica & . & + & + & + & . & . & . & . & . & . \\
\hline
\end{tabular}




\section{R. Gandullo et al. - Sintaxonomía de las comunidades de plantas vasculares}

territorio estudiado hemos reconocido el orden Phragmitetalia, que agrupa las comunidades propias de aguas poco o nada salinas y representado por una alianza: Phragmition, con dos subalianzas y por otra parte el orden Nasturtio officinalis - Glycerietalia fluitantis.

\section{* Phragmitetalia australis W. Koch $1926 \mathrm{em}$.} Pignatti 1954

Incluye a los carrizales instalados sobre suelos inundados casi permanentemente de agua dulce relativamente profunda. Forman la franja de helófitos más cercana al agua. Orden con biocenosis cosmopolitas.

+ Phragmition australis W. Koch 1926 nom. mut . Rivas Martínez y col. 2011

Comprende las asociaciones de carrizales y totorales de altura considerable, desarrolladas en suelos inundados casi permanentemente por aguas profundas; que se instalan en los bordes o en el interior de las zonas húmedas en las que el periodo de inundación es más prolongado, o en los cauces y bordes de arroyos, canales y ríos. Las comunidades de esta alianza son las que presentan una mayor exigencia de agua dentro de la clase Phragmitetea.

Es la única alianza en Europa en la que se pueden reconocer dos subalianzas: Phragmitenion australis y Bolboschoenenion maritimi, que se diferencian por la distinta capacidad que tienen de soportar la sequía (Rivas Martínez et al., 2002). Ambas, están representadas en la zona del Alto Valle de Río Negro.

++ Phragmitenion australis (Koch 1926) Rivas Martínez in Rivas Martínez, Costa, Castroviejo \& E. Valdés 1980

Subalianza que agrupa grandes helófitos rizomatosos dulceacuícolas (Molina, 1996), sensibles a los periodos de pérdida de agua (Rivas Martínez et al., 2002).

\section{Phragmitetum australis Soó 1927 nom. mut.} propos. Schmale 1939

Asociación muy extendida en la cuenca mediterránea (Šumberová et al., 2011). Comunidad del tipo sinantrópico-ruderal (vinculada a acciones antropogénicas) y humedales. Esta asociación se caracteriza fisonómicamente por cañas altas y densas de Phragmites australis, una gramínea con un amplio rango ecológico, que se presenta en humedales oligotróficos a eutróficos, ácidos a básicos e incluso salobres. Ocurre en zonas litorales de estanques, charcos aluviales, pozos inundados, zanjas, canales de drenaje, banquinas bajas con agua temporal, bordes de lodo y depresiones inundadas en tierras cultivables. Este tipo de vegetación se encuentra principalmente a una profundidad de agua de 10 a $50 \mathrm{~cm}$, pero también puede ocurrir en sitios que no están inundados en absoluto, pero con la presencia de la capa freática muy cerca de la superficie.

La composición florística es tan pobre que es difícil hacer distinciones, aunque ciertamente existen diferencias ecológicas, entre cuerpos de agua en Europa templada y mediterránea. En el Alto Valle, es poco común encontrar este tipo de asociación en sitios con agua superficial y periodos de agua permanente. Presenta entre una y cuatro especies (Fernández et al., 2018) y en la gran mayoría de los casos es monoespecífica. De acuerdo al dendrograma (Fig. 2), esta comunidad presentó similitud y afinidad con la asociación Typhetum angustifoliae. Esto hace suponer que la asociación Typho angustifoliae-Phragmitetum australis (Tüxen \& Preising, 1942) nom. nov. Rivas Martínez et al. (1991), podría estar representando la transición desde el Typhetum angustifoliae al Phragmitetum australis a medida que aumenta la eutrofia en el agua.

\section{Typhetum angustifoliae Pignatti 1953}

Comunidad dominada por Typha angustifolia que se encuentran en los cursos de agua mesotróficas a eutróficas (Pignatti, 1953), con fondos arcillosos y arenosos, a menudo con sedimentos orgánicos (Landucci, 2013), con agua de 20-60 cm de profundidad, inundada la mayor parte del año. Forma densos rodales a lo largo de los desagües rurales, zonas bajas inundadas, en canteras de piedra y áreas palustres de lagos artificiales. La asociación puede estar en contacto con el Phragmitetum australis típica de los hábitats de sucesión temprana con sustrato mineral en el fondo y generalmente desaparece a medida que se acumula el sedimento orgánico.

\section{Typhetum latifoliae Nowinski 1930}

Comunidad monoespecífica. Su presencia es ocasional en el Alto Valle (Jocou et al., 2018) en pequeños rodales de hasta $70 \%$ de 
cobertura con profundidades de agua de 15 a 20 $\mathrm{cm}$. Typha latifolia es la especie dominante y diagnóstica de esta comunidad presente en lugares fangosos, aguas turbias, $\mathrm{pH}$ ligeramente alcalino, moderadamente ricas en iones bicarbonato y ricas en nitrógeno inorgánico y fósforo. Se observó en varias oportunidades que Phragmites australis se encuentra lindante a la comunidad.

En el área investigada, las comunidades de Typha angustifolia y T. latifolia son estructural y ecológicamente muy similares. La diferencia radica en que $T$. latifolia se asocia, a diferencia de $T$. angustifolia, a mayores concentraciones de nitrógeno y fosfato orgánico en desagües claramente eutróficos. En el Alto Valle ambas especies se excluyen entre sí, nunca se observó que coexistan juntas en un sitio determinado. Typha latifolia prefiere lugares inundados someros la mayor parte del año, suelos ligeramente neutros a básicos y ricos en nutrientes, mientras que $T$. angustifolia es preferente de aguas oligotróficas más profundas y en diferentes tipos de sustratos.

\section{Typhetum domingensis Brullo et al. 1994}

Esta asociación está dominada por Typha domingensis, helófito de gran tamaño con distribución pantropical. Es común confundirla a campo con Typha angustifolia, por lo que es necesario realizar la diferenciación taxonómica en gabinete.

Asociación pobre en especies en su estado de madurez; sin embargo, al principio de temporada de crecimiento la diversidad es mayor. Recientemente se indicó la presencia de Epilobium hirsutum (Jocou \& Gandullo, 2018), como acompañante de esta asociación. Se desarrolla sobre suelos fangosos compactos, ligeramente salinos, ricos en materia orgánica, generalmente con sedimentos orgánicos con variable mineralización, que se presentan inundados y sometidos a sequía frecuente durante una época del año. Es considerado como un vicariante termófilo de Typhetum angustifoliae (Landucci, 2013). Se ubican por lo general en canales y desagües. Los totorales dominados por Typha domingensis están bastante difundidos en el Alto Valle debido a su adaptación a la contaminación acuática, la sequía y la salinización, suele desplazar frecuentemente a la asociación Typhetum angustifoliae cuando entra en competencia con la misma. Esto se refuerza con los estudios de Mufarrege (2012) en T. domingensis quien demostró que la especie presenta una alta eficiencia en la retención de metales y nutrientes y una elevada tolerancia a las condiciones de los efluentes tratados debido a su capacidad de adaptación fisiológica y morfológica.

\section{Typhetum subulatae ass. nov. (Tabla 1, Orden X y Tabla 5)}

Typus nomenclatorius associationis: Tabla 5, inv. 89

Diagnosis. De todas las comunidades de Typha es la menos difundida en el Alto Valle; fue descripta por primera vez por Fernández et al. (2018), restringida a desagües con escaso movimiento de agua, una profundidad entre $15-50 \mathrm{~cm}$, con agua transparente o levemente turbia, con porcentajes de cobertura de hasta el $85 \%$. Estructuralmente es similar a las otras asociaciones de Typha, por lo que el diagnóstico debe realizarse principalmente a través de la determinación taxonómica.

Especie característica: Typha subulata.

\section{Iridetum pseudacori Eggler 1933}

Esta asociación descripta para Europa y considerada adventicia en argentina, está caracterizada por la hierba palustre perenne Iris pseudacorus, citada recientemente para Río Negro por Jocou et al. (2018). En la zona de estudio forma grandes matas cespitosas aisladas en el interior y bordes de los desagües totalmente anegados, de modo más o menos permanente por aguas de corriente baja a moderada. Al ser una especie de carácter ornamental (Cheers, 1999) puede considerarse que ha sido introducida con ese propósito, sin embargo, se ha escapado de cultivo y naturalizado (Jocou et al., 2018).

Si bien no la hemos incluido en el esquema sintaxonómico por no contar con suficientes relevamientos, no descartamos que la misma se pueda establecer en la región del Alto Valle.

La posición sintaxonómica de la comunidad de Iris pseudacorus varía entre autores. Se optó por incluirla en el orden Phragmitetalia australis, por la presencia de elementos florísticos propios del orden como los helófitos Typha domingensis, Bolboschoenus maritimus y la herbácea Hydrocotyle ranunculoides.

++ Bolboschoenenion maritimi Rivas Martínez in Rivas Martínez, Costa, Castroviejo \& E. Valdés 1980 nom. mut. Rivas Martínez \& col. 2011 


\section{R. Gandullo et al. - Sintaxonomía de las comunidades de plantas vasculares}

Tabla 5. Typhetum subulatae Gandullo R., Fernández C. y Jocou A. I. Typus nomenclatorius associationis: inventario 89 (Gandullo, Fernández y Jocou) Prov. de Río Negro: Gral. Roca, zona rural. 18-03-2017 Densidad de especies: 1 (rango:1-3). Referencias= Localidades, Ce: Cervantes; CS: Cinco Saltos; A: Allen; Ma: Mainqué; GR: General Roca. Valores de cobertura, +: (<1); 1: (1-10); 2: (11-25); 3: (26-50); 4: (51-75); 5: $>75)$.

\begin{tabular}{|lcccccccccc|}
\hline Número de ordenamiento & 1 & 2 & 3 & 4 & 5 & 6 & 7 & 8 & 9 & 10 \\
Número de censo & 115 & 89 & 93 & 269 & 50 & 62 & 63 & 275 & 280 & 68 \\
Localidad & Ma & GR & Ce & Ma & CS & A & A & Ma & CS & A \\
Cobertura total \% & 95 & 85 & 85 & 80 & 70 & 70 & 70 & 65 & 60 & 30 \\
Número de especies & 3 & 1 & 1 & 1 & 1 & 2 & 2 & 1 & 1 & 2 \\
\hline Typhetum subulatae & & & & & & & & & \\
Typha subulata & 5 & 5 & 5 & 5 & 4 & 4 & 4 & 4 & 3 & 2 \\
Schoenoplectus californicus var. californicus & 1 &. &. &. &. & + & 1 &. &. &. \\
Epilobium ciliatum ssp. ciliatum & + &. &. &. &. &. &. &. &. &. \\
Juncus microcephalus &. &. &. &. &. &. &. &. &. \\
\hline
\end{tabular}

Subalianza que une las asociaciones junceiformes dulceacuícolas de aguas someras poco sensibles a la desecación.

\section{Bolboschoenetum maritimi Br. Bl. 1931}

Comunidad muy pobre florísticamente, dominada por Bolboschoenus maritimus, que se desarrolla sobre una gran variedad de suelos periódicamente inundados por aguas dulces, en ocasiones ricas en nutrientes.

Bolboschoenus maritimus demostró ser tolerante a la salinidad y crecer en desagües salinizados. En el Alto Valle de Río Negro, la asociación está escasamente representada y restringida a dos localidades. Presenta altas coberturas, entre $80 \%$ y $100 \%$, y con plantas de altura media, hasta un metro.

\section{* Nasturtio officinalis - Glycerietalia fluitantis} Pignatti 1953

En este orden se incluyen comunidades dulceacuícolas de helófitos latifolios decumbentes o graminícolas y graminiformes, erguidos, flexibles o flotantes, de talla media o pequeña; propios de aguas estancadas y fluyentes, poco profundas, ricas en nutrientes nitrogenados, sometidas a desecaciones estivales por lo general prolongadas. Presenta distribución cosmopolita. Para el área de estudio, se reconoce la siguiente alianza con dos asociaciones y una comunidad.
+ Apion nodiflori Segal in Westhoff \& den Held 1969

Vegetación palustre de ribera, representa las comunidades semisumergidas de márgenes de cursos de agua dulce permanentes, más o menos de corriente rápida y someras, desde mesotróficos a eutróficos. En algunos casos también puede colonizar las partes internas de los canales y desagües poco profundos y de corrientes lentas. Especies frecuentes: Nasturtium officinalis y Veronica anagallis-aquatica. Se optó por incluir las asociaciones y la comunidad encontradas en la presente alianza por las condiciones del biotopo característico donde crecen. Es plausible que esto se deba a una vicarianza de las sintaxa, cuyas especies involucradas son Apium nodiflorum y A. graveolens.

1. Nasturtietum officinalis (Seibert 1962) Oberd. et al. 1967

Asociación constituida por hierbas acuosas y tiernas, siempre verdes, dominadas por Nasturtium officinale, taxón frecuente en medios acuáticos, es rara en los lugares de encharcamiento temporal. Coloniza los bordes de cursos de agua dulce más o menos eutrofizados, poco profundos y semipermanentes, sobre sustratos arcillosos, limosos o incluso, fangosos. Se presenta ampliamente extendida en la mayor parte de los desagües de la zona con agua tranquilas o corrientes. Acompañan especies natantes como Lemna gibba, Azolla filiculoides y emergentes 
como Hydrocotyle ranunculoides, Ludwigia grandiflora y Typha domingensis. Cabe destacar que la cenosistemática europea incluye la presente asociación en la alianza Glycerio - Sparganion Braun-Blanquet et Sissingh in Boer 1942, por el momento se considera más oportuno incluirla en la presente alianza por su afinidad florística, hasta que se amplíen los estudios de la misma.

\section{Hydrocotyletum ranunculoidis Corbetta \& Lorenzoni 1976}

La especie dominante y diferencial de esta asociación es Hydrocotyle ranunculoides. Es preferente de ambientes eutrofizados en arroyos y desagües de aguas loticas, someras, de escaso movimiento y altamente contaminadas con materia orgánica, tanto de origen industrial como rural (Fernández et al., 2018). También suele estar presente en suelos saturados no inundados, en los márgenes poco profundos de los desagües. Cuando baja el nivel del agua del desagüe las hojas se vuelven amarillas y mueren. Representa la transición entre las plantas acuáticas y las mesófitas. $H$. ranunculoides presenta raíces y rizomas dotados de abundante aerénquima como mecanismo de adaptación a la disminución en la disponibilidad de oxígeno bajo el agua.

Hydrocotyletum ranunculoidis fue descrito por Ramírez \& Beck (1981) en lagunas altiplánicas de Bolivia. Según San Martín et al. (2001) es una asociación muy próxima a Nasturtio - Hydrocotyletum ranunculoidis y a Hydrocotylo - Callitrichetum stagnalis descrito por Barrera y Ramírez (1986) y caracterizada como una comunidad de aguas contaminadas con residuos domésticos (Finot y Ramírez, 1996). San Martín et al. (2001) sugieren una revisión, ya que al parecer existe mucha sinonimia de la misma comunidad.

3. Comunidad de Ludwigia grandiflora ssp. hexapetala

Vegetación helofítica perenne, caracterizada por Ludwigia grandiflora que presenta dimorfismo foliar (hojas natantes y aéreas), con estatus de nativa para nuestro país.

Ocupa amplio rango de hábitats desde las orillas de sectores fangosos con escaso escurrimiento hasta el centro del cuerpo de agua de los desagües. Tolera diferentes niveles de nutrientes, tipos de sustratos y calidad de aguas. Comunidad que reduce el $\mathrm{pH}$ del agua y produce una desoxigenación de la misma, por la gran captación de oxígeno a través de sus raíces adventicias, lo que conduce a un daño negativo para la fauna, la reducción de la germinación y crecimiento de otras especies de plantas.

$\mathrm{Su}$ alta cobertura y biomasa es la causante de un aumento de sedimentos, de la reducción de la penetración de la luz, de la disminución de las escorrentías y de la alteración de la circulación de las aguas, factores que generan efectos negativos en la biodiversidad y en calidad del agua. Por el momento consideramos oportuno mantenerla en el rango de comunidad de Ludwigia grandiflora ssp. hexapetala hasta ampliar los estudios de la misma.

\section{Consideraciones sintaxonómicas}

La presente investigación contribuye al Prodromus sinsistemático argentino en el ordenamiento sintaxonómico de la vegetación acuática y se citan por primera vez cuatro (4) nuevas asociaciones, con una breve descripción ecológica, para los ambientes lóticos artificiales de Argentina. Además, se destacan once (11) asociaciones con potencial bioindicador de eutrofización, establecidas en la red de drenaje del Alto Valle.

\section{Conclusiones}

Se establecieron veintiocho categorías sintaxonómicas: tres clases, cinco ordenes, cinco alianzas, dos subalianzas y trece asociaciones para el sistema de drenaje del Alto Valle, ordenadas en función de tres hábitos de crecimiento: acuáticas flotantes, sumergidas y emergentes.

Las especies diferenciales de la vegetación acuática del Alto Valle son: Azolla filiculoides, Lemna gibba, Stuckenia pectinata, Myriophyllum aquaticum, Elodea callitrichoides, Phragmites australis, Typha angustifolia, T. latifolia, T. domingensis, T. subulata, Bolboschoenus maritimus, Nasturtium officinale y Hydrocotyle ranunculoides.

Se determinaron cuatro nuevas asociaciones vegetales acuáticas para la ciencia: Azolletum filiculoidis, Azollo filiculoides - Lemnetum gibbae, Elodeetum callitrichoidis y Typhetum subulatae incluidas en las clases Lemnetea minoris, Potametea y Magnocari elatae - Phragmitetea australis de acuerdo al esquema sintaxonómico planteado. 


\section{R. Gandullo et al. - Sintaxonomía de las comunidades de plantas vasculares}

El $80 \%$ de las asociaciones poseen potencial bioindicador de eutrofización en el sistema de drenaje del Alto Valle de Río Negro.

\section{Contribución de los aUtores}

Todos los autores han contribuido conjuntamente y en partes iguales tanto en la colecta de datos como en la redacción del manuscrito.

\section{Agradecimientos}

Los autores agradecen a los revisores anónimos por sus valiosas sugerencias y comentarios enriquecedores sobre este artículo. El presente trabajo se financió con fondos del Proyecto PI A129 de la Secretaría de Ciencia y Técnica de la Universidad Nacional del Comahue.

\section{Bibliografía}

AKASAKA, M. \& N. TAKAMURA. 2011. The relative importance of dispersal and the local environment for species richness in two aquatic plant growth forms. Oikos 120: 38-46. https://doi.org/10.1111/j.1600-0706.2010.18497.x

ALBA TERCEDOR, J. 1996. Macroinvertebrados acuáticos y calidad de las aguas de los ríos. Actas del IV Simposio del agua en Andalucia (SIAGA) 2: 203-213.

ALCARAZ ARIZA, F. J. 2013. Fundamentos de la clasificación de la vegetación. Universidad de Murcia, España. Disponible en: https://www.um.es/docencia/ geobotanica/ficheros/tema10.pdf [Consulta: 15 mayo 2019].

AROCENA, R. \& N. MAZZEO. 1994. Aquatic macrophytes of an urban river in Uruguay: The relation to water quality. Revista Biol. Trop. 42: 723-728.

AROCENA, R., D. FABIAN \& J. CLEMENTE. 2000. Las causas naturales versus la contaminación orgánica como factores estructuradores del zoobentos en tres afluentes de una laguna costera. Limnetica 18: 99-113.

AVENA, G. C., C. BLASI \& A. SCOPPOLA. 1980. Indagini ecologiche e fitogeografiche sulle zone umide interne del Lazio. 1: Relazione sullo stato chimico-fisico delle acque e le comunità macrofitiche della Bonifica Pontina. Ann. Bot. (Rome) 39: 31-89.
BALDONI, M. \& E. BIONDI. 1993. La vegetazione del medio e basso corso del fiume Esino (Marche - Italia Centrale). Stud. Bot. 11: 209-257.

BARRERA, J. \& C. RAMÍREZ. 1986. Origen, características y aprovechamiento de los bañados del sur de Chile. Versiones Abreviadas II Encuentro Cientifico sobre el Medio Ambiente Chileno, Talca, 1: 52-56.

BEZIC, C., A. DALL'ARMELLINA, F. HORNE, O. GAJARDO, L. AVILÉS \& S. CAÑÓN. 2004. Distribución y abundancia de macrófitas sumergidas en el embalse de Casa de Piedra. Pilquen 6: 1-7.

CASTRO GONZÁLEZ, V. 2015. Flora y vegetación de las lagunas y humedales del sector castellanoduriense en la provincia de León. Bases para su conservación y gestión sostenible. Tesis Doctoral. Departamento de Biodiversidad y Gestión Ambiental (Área de Botánica) Universidad de León. España.

CESCHIN, S., V. ZUCCARELLO \& G. CANEVA. 2010. Role of macrophyte communities as bioindicators of water quality: Application on the Tiber River basin (Italy). Pl. Biosyst. 144: 528-536. https://doi.org/10.1080/11263500903429221

CHEERS, G. 1999. Botanica, the illustrated A-Z of over 10,000 garden plants and how to cultivate them, 3ra ed. Könemann, Milsons Point.

CONTICELLO, L., B. CERAZO \& A. BUSTAMANTE. 2002. Dinámica de comunidades hidrófilas asociadas a canales de riego en el Alto Valle de Río Negro (Argentina). Gayana, Bot. 59: 13-20. https://doi.org/10.4067/S0717-66432002000100003

DIEKMANN, M. 2003. Species indicator values as an important tool in applied plant ecology-a review. Basic Appl. Ecol. 4: 493-506. https://doi.org/10.1078/1439-1791-00185

ELLENBERG, H. 1950. Landwirtschfliche Pflansensociologie. I. Unkraut-Gemeinschaften als Zeiger für Klima und Boden. Eugen Ulmer, Stuttgart.

ELLENBERG, H. 1974. Indicator values of vascular plants in Central Europe. Scripta Geobot. 9:1-17.

ELLENBERG, H., H. E. WEBER, R. DULL, V. WIRTH, W. WERNER \& D. PAULIBEN. 1992. Zeigerwerte von Pflanzen in Mitteleuropa. Scripta Geobot. 18: $1-258$.

FERNÁNDEZ, C., A. I. JOCOU \& R. GANDULLO. 2018. Vegetación acuática bioindicadora de eutrofización del Alto Valle de Rio Negro (Argentina). Ernstia 28: 45-93.

FERRERAS, C. \& C. FIDALGO. 1999. Biogeografia y Edafogeografia. Síntesis, Madrid. 
FINOT, V. L. \& C. RAMÍREZ. 1996. Fitosociología de la vegetación ruderal de la ciudad de Valdivia (X RegiónChile). 1. Vegetación nitrófila. Ediciones Universidad de Salamanca. Stud. Bot. 15: 159-170.

GONZALÉZ LÓPEZ, C. A. 2005. Biodiversidad vegetal acuática de los humedales del río Chepu (Chiloé, Región de Los Lagos, Chile). Tesis de grado. Universidad Austral de Chile. Valdivia, Chile. 113 pp.

HRUŠKA, K. 1987. The role of natural nitrophilous vegetation in the evaluation of the quality of the environment. Strasbourg. Coll. Phytosoc. 15: 108-116.

IBODA. Continuamente actualizado. Base de datos. Flora del cono Sur. Instituto de Botánica Darwinion. Buenos Aires, Argentina [online]. Disponible en: http://www2.darwin.edu.ar. [Acceso 15 agosto 2019].

JOCOU, A. I., C. FERNÁNDEZ \& R. GANDULLO, 2018. Macrófitas acuáticas vasculares del sistema de drenaje del Alto Valle de Río Negro, Patagonia (Argentina). Revista del Museo de La Plata 3: 296308. https://doi.org/10.24215/25456377e060

JOCOU, A. I. \& GANDULLO, R. 2018. Primer registro de Epilobium hirsutum (Onagraceae) para la flora Argentina. Darwiniana, n.s. 6: 99-107. https://doi.org/10.14522/darwiniana.2018.61.784

KNAPP, R. 1984. Considerations on quantitative parameters and qualitative attributes in vegetation and phytosociological relevés. In: KNAPP, R. (ed.), Sampling methods and taxon analysis in vegetation science, pp. 77-100. Dr. W. Junk Publisher, La Haya.

KOLLMANN, J. \& A. FISCHER. 2003. Vegetation as indicator for habitat quality. Basic Appl. Ecol. 4: 489-491. https://doi.org/10.1078/1439-1791-00209

LANDOLT, E. 1980. Key to the determination of taxa within the family of Lemnaceae. Veröff. Geobot. Inst. E. T. H. Stiftung Rübel Zürich. 70: $13-21$

LANDOLT, E. 1996. Flora Fanerogámica Argentina. Lemnaceae. ProFlora CONICET, Argentina.

LANDOLT, E. 1999. Pleustonic communities with Lemnaceae in South America. Appl. Veg. Sci. 2: 7-16. https://doi.org/10.2307/1478876

LANDUCCI, F., D. GIGANTE \& R. VENANZONI. 2011. An application of the Cocktail method for the classification of the hydrophytic vegetation at Lake Trasimeno (Central Italy). Fitosociologia 48: 3-22.

LANDUCCI, F. 2013. Wetland vegetation of the class Phragmito-Magnocaricetea in central Italy. Phytocoenologia 43: 67-100. https://doi.org/10.1127/0340-269X/2013/0043-0545
LOZECO, C. V. 2014. Desarrollo de un esquema de gestión integrada para los colectores de drenaje de la ciudad de Cipolletti (Río Negro, Argentina). Tesis de Maestría. Universidad Nacional del Litoral. Santa Fe, Argentina. 224 pp.

MARTÍNEZ CARRETERO, E., A. M. FAGGI, J. L. FONTANA, P. ACEÑOLAZA, R. GANDULLO, M. CABIDO, D. IRIART, D. PRADO, F. A. ROIG \& U. ESKUCHE. 2016. Prodromus Sinsistemático de la República Argentina y una breve introducción a los estudios fitosociológicos. Bol. Soc. Argent. Bot. 51: 469-549.

MC CUNE, B. \& M. J. MEFFORD. 1999. "PCORD” Multivariate analysis of ecological data, version 4. MJM Software design, Oregon.

MOLINA, J. A. 1996. Sobre la vegetación de los humedales de la Península Ibérica (PhragmitiMagnocaricetea). Lazaroa 16: 27-88.

MUFARREGE, M. M. 2012. Tolerancia y eficiencia de Typha domingensis Pers. en la retención de metales y nutrientes de efluentes industriales. Tesis para optar el grado de Dr. Facultad de Ingeniería Química, Universidad Nacional del Litoral. Santa Fe, Argentina.

MÜLLER-DOMBOIS, D. \& H. ELLENBERG. 1974. Aims and methods of vegetation ecology. John Wiley \& Sons, New York.

OBERDORFER, E. 1960. Pflanzensoziologishe Studien in Chile: Ein Vergleich mit Europa. Fl. Veg. Mundi 2: $1-208$

ORLOCI, L. 1967. An agglomerative method for the classification of plant communities. J. Ecol. 55: 193206. https://doi.org/10.2307/2257725

PIGNATTI, S. 1953. Introduzione allo studio fitosociologico della pianura veneta orientale. Atti Ist. Bot. Univ. Lab. Critt. Pavia 11: 92-258.

RAMÍREZ, C. \& S. BECK. 1981. Makrophytische Vegetation und Flora in Gewässern der Umgebung von La Paz, Bolivien. Arch. Hydrobiol. 91: 82-100.

RAMÍREZ, C.\& J. SAN MARTÍN. 1984. Hydrophylous vegetation of a coastal lagoon in Central Chile. Int. J. Ecol. Environm. Sci. 10: 93-100.

RAMÍREZ, C., J. SAN MARTÍN, C. SAN MARTÍN \& D. CONTRERAS. 1987. Estudio florístico y vegetacional de la laguna El Peral, Quinta Región de Chile. Rev. Geogr. Valpso. 18: 105-120.

RAMÍREZ, C., C. SAN MARTIIN \& J. SAN MARTÍN. 2004. Colmatación por macrófitos del complejo lacustre Vichuquén(VII Región, Chile) y clave de determinación. Revista Geográfica de Chile Terra Australis 49:179-196. 


\section{R. Gandullo et al. - Sintaxonomía de las comunidades de plantas vasculares}

RAMÍREZ, C. \& C. SAN MARTÍN. 2006. Diversidad Macrofitas chilenas. En: Vila, I., A. Veloso, R. Schlatter, C. Ramírez (eds.), Macrófitas y vertebradps de los sistemas límnicos de Chile, pp. 21-72. Ed. Universitaria, Santiago de Chile.

RAMÍREZ, C. \& M. ÁLVAREZ. 2012. Flora y vegetación hidrófila de los humedales costeros de Chile. En: Fariña, J. M. \& A. Camaño (eds.), Humedales costeros de Chile: Aportes científicos a su gestión sustentable, pp. 101-145. Ediciones Universidad Católica de Chile, Santiago de Chile.

RAMÍREZ, C., J. M. FARIÑA, A. CAMAÑO, D. CONTRERAS, C. SAN MARTÍN, J. VARAS, O. VIDAL \& Y. PÉREZ. 2014. Estructura y clasificación de la vegetación actual y potencial del humedal "Ciénagas del Name" en Chile Central: Un estudio de la oferta de hábitats. Chilean J. Agric. Anim. Sci. 30: 29-44.

RIVAS MARTÍNEZ, S. 1975. Datos ecológicos sobre la vegetación acuática continental. Anal. Inst. Bot. Cavanilles 32: 199-205.

RIVAS MARTÍNEZ, S. 1987. Memoria del mapa de series de vegetación de España. Ministerio de Agricultura, Pesca y Alimentación, Madrid.

RIVAS MARTÍNEZ, S. 1995. Clasificación bioclimática de la Tierra (Bioclimatic Classification System of the World). Folia Bot. Matritensis 16: 1-25

RIVAS MARTÍNEZ, S., T. DÍAZ, F. FERNÁNDEZ GONZÁLEZ, J. IZCO, J. LOIDI, M. LOUSÁ \& A. PENAS. 2002. Vascular Plant Communities of Spain and Portugal. Itin. Geobot. 15: 5-432.

RODWELL, J. S. 1991. British plant communities, vol. 2: Mires and heaths. Cambridge University Press, Cambridge.

RODWELL, J. S. 1995. British plant communities, vol. 4: Aquatic communities, swamps and tall-herb Fens. Cambridge University Press, Cambridge.

ROIG, F. A. 1973. El cuadro fitosociológico en el estudio de la vegetación. Deserta. 4: 45-67.

SAN MARTÍN, C., R. MEDINA, P. OJEDA\& C. RAMÍREZ. 1993. La biodiversidad vegetacional del Santuario de la Naturaleza "Río Cruces" (Valdivia, Chile). Acta Bot. Malac. 18: 259- 279.

SAN MARTÍN, C., C. RAMÍREZ \& M. VERDUGO. 1998. Sinecología de las praderas húmedas de junquillo en el centro-sur de Chile. Anales de la Sociedad Chilena de Ciencias Geográficas 1: 87-94.
SAN MARTÍN, C., C. RAMIREZ, J. SAN MARTÍN \& R. VILLASEÑOR. 2001. Flora y vegetación del estero Reñaca (V Región, Chile). Gayana, Bot. 58: 31-46. https://doi.org/10.4067/S0717-66432001000100004

SAN MARTÍN, C., C. RAMÍREZ \& M. ÁlVAREZ. 2003. Macrófitos como bioindicadores para caracterizar ambientes dulceacuícolas. Rev. Geogr. Valpso. 34: 243-253.

SCOPPOLA, A. 1982. Considerations nouvelles sur les vegetations des Lemnetea minoris (R. Tx. 1955) em. A. Schwabe et R. Tx. 1981 et contribution a l'etude de cette classe en Italie centrale. Doc. Phytosoc. 6: $1-130$.

SCULTHORPE, C. D. 1967. The Biology of Aquatic Vascular Plants. St. Martin's, New York

ŠTĚPÁNKOVÁ J., CHRTEK J. \& Z. KAPLAN. 2010. Lemna minor L. - okřehek menší. In: Štěpánková J., Chrtek J., Kaplan Z. (eds), Květena České republiky, pp. 289-290. Academia, Praha.

ŠUMBEROVÁ, K. 2011. Vegetace volněplovoucích vodních rostlin (Lemnetea), Vegetation of freefloating aquatic plants. In: CHYTRÝ, M. (ed.), Vegetation of the Czech Republic 3, Aquatic and wetland vegetation, pp. 43-99. Academia, Praha.

ŠUMBEROVÁ, K., P. HÁJKOVÁ, M. CHYTRÝ, Z. HROUdOVÁ, J. SÁDLO, M. HÁJEK, R. HRIVNÁK, J. NAVRÁTILOVA, P. HANÁKOVÁ, L. EKRT \& E. EKRTOVÁ. 2011. Vegetace rákosin a vysokých ostřic (Phragmito-Magno-Caricetea). Marchland vegetation. In: Chytrý M. (ed.), Vegetace České republiky 3. Vodní a mokřadní vegetace, pp. 385-580. Academia, Praha.

VENANZONI, R. \& D. GIGANTE. 2000. Contributo alla conoscenza della vegetazione degli ambienti umidi dell'Umbria. Fitosociologia 37: 13-63.

VENANZONI, R., D. GIGANTE, L. MONTAGNOLI \& M. FRATTEGIANI. 2006. Habitat e specie della Direttiva 92/43/CEE ed altri aspetti di rilevanza naturalistica al Lago Trasimeno. AP\&elle, Perugia.

WARD, J. H. 1963. Hierarchical grouping to optimize an objective function. J. Am. Statist. Ass. 58: 236-244. https://doi.org/10.1080/01621459.1963.10500845

WEBER, H. E., J. MORAVEC \& J. P. THEURILLAT. 2000. International Code of Phytosociological Nomenclature. J. Veg. Sci. 11: 739-768. https://doi.org/10.2307/3236580 
